\section{PO 8564 WHAT IS CLINICAL TRIAL QUALITY? A QUALITATIVE STUDY BASED ON INTERVIEWS WITH DIFFERENT STAKEHOLDERS CONDUCTING CLINICAL TRIALS IN SUB- SAHARAN AFRICA}

1,2Angela Lazarova, ${ }^{2}$ Claudia Fuchs, ${ }^{1,2}$ Christian Burri. 'S Swiss Tropical and Public Health
Institute, Basel, Switzerland; ' 2 University of Basel, Basel, Switzerland

10.1136/bmjgh-2019-EDC.141

Background There is no unified, broad definition for quality in clinical trials (CTs). Besides the explicit quality requirements in international guidelines and national legislation, however, there are broader factors to consider, including specific setting-related aspects influencing quality needs, quality perception and local implementation of guidelines. Our objective was to identify these factors from a resource-limited settings perspective (in this case, sub-Saharan Africa).

Methods In March-April 2018, we conducted a qualitative study based on semi-structured interviews with participants from three stakeholder groups (monitors, sponsors, and investigators) conducting CTs in sub-Saharan Africa. We identified the interviewees either through CT registry platforms, a web search or by reference. We aimed to include 10-20 participants per stakeholder group. After consent, the interviews were held in person (via Skype or telephone), recorded, and transcribed verbatim. The interview questionnaire addressed a CT quality definition and quality factors during the CT process. We performed the analysis using the framework method.

Results So far, we included 21 participants (17 investigators, two sponsors, two monitors). Eight (8) (from sub-Saharan Africa) and 13 (not from sub-Saharan Africa) who contributed to CTs in 19 different countries in sub-Saharan Africa. Quality definitions mentioned so far were variable. A repeated statement was that the quality definition should be broad and include a system of multiple aspects and layers. We will interview more experts with sponsor and monitor experience in May 2018 and elaborate these quality aspects and layers. We will discuss these results with regards to a comprehensive quality framework for CTs currently under development for Northern countries by another Swiss research team.

Conclusion CT quality was perceived in variable ways, as was the relevance of the aspects across different CT steps. Structuring the multifaceted layers of CT quality will facilitate appropriate and efficient CT quality management in sub-Saharan Africa.

\section{PO 8565 PREVALENCE AND CLINICAL SIGNIFICANCE OF RESPIRATORY VIRUSES AND BACTERIA DETECTED IN TUBERCULOSIS PATIENTS COMPARED TO HOUSEHOLD CONTACT CONTROLS IN TANZANIA}

\begin{abstract}
${ }^{1}$ Francis Mhimbira* ${ }^{*}$ 1,2,3 Jerry Hella, ${ }^{1}$ Hellen Hiza, ${ }^{1}$ Emmanuel Mbuba, ${ }^{4}$ Magreth Chiryamkubi, ${ }^{2,3}$ Sebastien Gagneux, ${ }^{5}$ Lukas Fenner. 'Ifakara Health Institute, Tanzania; ${ }^{2}$ Swiss Tropical and Public Health Institute, Basel, Switzerland; ${ }^{3}$ University of Basel, Switzerland; " Ministry of Health, Community Development, Gender, Elderly and Children, Dar es Salaam Tanzania; ${ }^{5}$ Institute of Social and Preventive Medicine, University of Bern, Switzerland
\end{abstract}

10.1136/bmjgh-2019-EDC.142

Background The study aim is to describe the prevalence of respiratory pathogens in tuberculosis (TB) patients and in their household contact controls, and to determine the clinical significance of respiratory pathogens in TB patients.

Methods We studied 489 smear-positive adult TB patients and 305 household contact controls without TB with nasopharyngeal swab samples within an ongoing prospective cohort study in Dar es Salaam, Tanzania, between 2013 and 2015. We used multiplex real-time PCR to detect 16 respiratory viruses and seven bacterial pathogens from nasopharyngeal swabs.

Results The median age of the study participants was 33 years; 61\% (484/794) were men, and 21\% (168/794) were HIV-positive. TB patients had a higher prevalence of HIV $(28.6 \% ; 140 / 489)$ than controls $(9.2 \% ; 28 / 305)$. Overall prevalence of respiratory viral pathogens was 20.4\% (160/794; 95\% CI $17.7 \%-23.3 \%)$ and of bacterial pathogens $38.2 \%$ (303/794; 95\% CI 34.9\%-41.6\%). TB patients and controls did not differ in the prevalence of respiratory viruses (Odds Ratio [OR] 1.00, 95\% CI 0.71-1.44), but respiratory bacteria were less frequently detected in TB patients (OR 0.70, 95\% CI 0.53-0.94). TB patients with both respiratory viruses and respiratory bacteria were likely to have more severe disease (adjusted OR [aOR] 1.6, 95\% CI 1.1-2.4; p 0.011). TB patients with respiratory viruses tended to have more frequent lung cavitations (aOR 1.6, 95\% CI 0.93-2.7; p 0.089).

Conclusion Respiratory viruses are common for both TB patients and household controls. TB patients may present with more severe TB disease, particularly when they are co-infected with both bacteria and viruses.

\section{PO 8567 ASSOCIATION OF POLYMORPHISM RS 73885319 OF THE APOL1 GENE AND RESISTANCE/SUSCEPTIBILITY TO TRYPANOSOMA BRUCEI GAMBIENSE}

Mwamba Miaka Erick, Veerle Lejon, Hamidou Ilboudou, Philippe Solano. Institut de développement et de recherche, Marseille, France

\subsection{6/bmjgh-2019-EDC.143}

Background In Central Africa, human African trypanosomiasis (HAT) or sleeping sickness is caused by Trypanosoma brucei gambiense (T.b gambiense). Classically, the disease is characterised by an early haemolymphatic phase (stage 1) followed by a meningo-encephalitic phase (stage 2) leading to neurological disorders and death if left untreated. However, field observations suggest that infection by T. $b$ gambiense may result in a great diversity of clinical outcomes ranging from rapid progressions into stage 2, to asymptomatic infections that can last for years or even spontaneous cure in the absence of treatment.

The determinants of this clinical diversity are not known but might have their origin both in the parasite (genetic variability) and in the host (individual susceptibility to disease). This study in the Democratic Republic of the Congo aimed at examining the association between the rs73885319 polymorphism of the APOL1 gene and resistance/susceptibility to T. $b$ gambiense. We genotyped the APOL1 gene polymorphism in a total of 257 people comprised of 90 patients, 119 endemic controls and 48 seropositives. The analysis of the results has not shown any significant differences between HAT patients, controls and seropositives. Our results seem to suggest that the $G$ allele of the rs 73885319 polymorphism of the APOL1 gene is not associated to resistance or susceptibility to infection. 